\title{
Measurement of Coherent Undulator Radiation of Compact Terahertz Radiation Source at Kyoto University
}

\author{
Sikharin Suphakul*, Heishun Zen, Toshiteru Kii and Hideaki Ohgaki
}

Institute of Advanced Energy, Kyoto University, Japan

\begin{abstract}
An accelerator based compact terahertz radiation source has been successfully developed at Institute of Advanced Energy, Kyoto University. The system is very compact with the total length of $3.7 \mathrm{~m}$. The radiations are generated by injecting a short electron bunch to a $0.7 \mathrm{~m}$ long 10-period planar Halbach type undulator. The radiations have a high degree of phase coherence or called coherent undulator radiation. The frequencies are tunable in the range from 0.17 to $0.55 \mathrm{THz}$. The electron source is a 1.6 cells BNL type photocathode RF gun which provides the beam with energy of $4.6 \mathrm{MeV}$. The beams are compressed in longitudinal by a magnetic chicane bunch compressor. The first light was generated in Aug 2016. The results of the measurement of the light properties are presented in this paper.
\end{abstract}

Keywords

Undulator radiation, Coherent radiation, Terahertz

\section{Introduction}

Over past few decades, the studies based on Terahertz $(\mathrm{THz})$ frequency electromagnetic radiation or terahertz science gain great interests in various fields from fundamental researches to industrial applications. The frequency of $\mathrm{THz}$ radiation is in the far infrared spectral range (approximately 0.1 to $10 \mathrm{THz}$ ) which corresponds to the intermolecular vibration [1]. THz radiation allows us to extend the study of the molecular dynamics beyond conventional infrared radiation techniques leading to new information or methods of a material analysis, a medical diagnostic, a non-destructive inspection or a security, etc. However, the $\mathrm{THz}$ radiation is difficult to generate by conventional devices. Therefore, the development of the $\mathrm{THz}$ source is one of the main challenges in $\mathrm{THz}$ science, especially in the high power range.

An accelerator-based $\mathrm{THz}$ radiation source is a kind of sources that can provide significantly high power, tunable frequency and narrow bandwidth. There are many accelerator-based $\mathrm{THz}$ radiation sources operating worldwide, such as, at JLAB [2], DESY [3], SLAC [4]. However, these systems are large-scale facilities that require high cost for the construction and the operation. Over past decade, a small-scale accelerator system has been proven that it has the potential to achieve high power and continuous tunability in the $\mathrm{THz}$ radiation regime. The system employs bunched electron beams whose bunch length comparable to the radiation wavelength injecting to a short undulator. The radiation from the undulator has a high degree of phase coherence or called Coherent Undulator Radiation (CUR). There are a number of systems that have been developed, for examples the compact systems at ENEA $(0.4-0.7 \mathrm{THz})$ [5] and Peking Univ. (0.24-0.42 THz) [6] etc.

We also have been developed accelerator-based compact $\mathrm{THz}$ radiation source at Institute of Advanced Energy, Kyoto University. The system is able to produce a high power tunable $\mathrm{THz}$ CUR by injecting a short electron bunch to a short planar undulator. The system is compact with the total length of $3.7 \mathrm{~m}$ and shares the building and the RF power source with Kyoto University Free-Electron Laser (KU-FEL) [7]. The first photoelectron beam was generated from a 1.6 cells S-band BNLtype photocathode RF gun in April 2015 [8]. The mag-

*Corresponding author: Sikharin Suphakul, Institute of Advanced Energy, Kyoto University, Gokasho, Uji, Kyoto, 611-0011, Japan, E-mail: sukarin.supakun.78z@st.kyoto-u.ac.jp

Received: April 29, 2017: Accepted: June 29, 2017: Published: July 01, 2017

Copyright: () 2017 Suphakul S, et al. This is an open-access article distributed under the terms of the Creative Commons Attribution License, which permits unrestricted use, distribution, and reproduction in any medium, provided the original author and source are credited. 
netic chicane bunch compressor was installed in March 2016 and the compressed electron bunch characteristic was investigated by the coherent transition radiation technique in April 2016 [9]. The undulator was installed in July 2016. The first THz CUR was successfully generated in August 2016. The characteristic of the radiation were measured by two small-size uncooled detectors and the spectra were analyzed by an in-air Michelson interferometer. This paper presents the details, results and discussions of the measurement of the first $\mathrm{THz}$ CUR. However, the absolute values of the intensity were not calculated because the precise calibration of the measurement system is still underway.

\section{Coherent Undulator Radiation}

Undulator is a device which generates a periodic magnetic field to drive the electron in a sinusoidal trajectory. During electrons travel through an undulator, electrons emit the radiations in the forward direction as shown in Figure 1 and the radiation wavelength $(\lambda)$ can be determined by

$$
\lambda=\frac{\lambda_{u}}{2 \gamma^{2}}\left(1+\frac{K^{2}}{2}+\gamma^{2} \theta^{2}\right)
$$

Where $\lambda_{u}$ is the undulator period length, $\gamma$ is the Lorentz factor, $\theta$ is the observation angle from the beam direction, $\mathrm{K}$ is the undulator parameter, $\mathrm{K}=\mathrm{eB}_{0} / \mathrm{m}_{\mathrm{e}} \mathrm{ck}_{\mathrm{u}}$ $\mathrm{m}_{\mathrm{e}}$ is the charge of an electron, $B_{0}$ is the peak magnetic field, me is the rest mass of electron and $c$ is the speed of light and $k_{\mathrm{u}}$ is the undulator wave number, $2 \pi / \lambda_{u}$. The radiations concentrate in a narrow cone with the opening angle of $K / \gamma$. The Coherent Undulator Radiation (CUR) can be obtained when the electron bunch length is comparable to the radiation wavelength. The total field of CUR $\left(\mathrm{E}_{\text {tot }}(\omega)\right)$ at frequency $\omega$ can be determined by a sum of the emitted fields from each electron [10] as

$$
E_{\text {tot }}(\omega)=N^{2} f(\omega) E_{1 e}(\omega)
$$

Where $N$ is the number of electrons, $f(\omega)$ is the Fourier transform of the electron distribution or called the form factor and $E_{1 e}$ is the field of a single electron. The $f(\omega)$ in the longitudinal direction can be calculated by

$$
f(\omega)=\int s(z) e^{i k z} d z
$$

Where $s(z)$ is the normalized electron bunch distribution in longitudinal direction, $z$ is the electron position in longitudinal direction and $k$ is the wave number of the radiation, $k=\omega / c=2 \pi / \lambda$. The total intensity of the CUR $(I(\omega))$ equal to the total field square, $I(\omega) \alpha\left|E_{\text {tot }}(\omega)\right|^{2}$. To obtain a high CUR intensity, we employed the bunch compressor to compress electron bunch longitudinally to a shorter bunch length resulting in the enhancement of the form factor and the CUR intensity. The schematic diagram of the bunch compression in longitudinal is shown in the Figure 2.

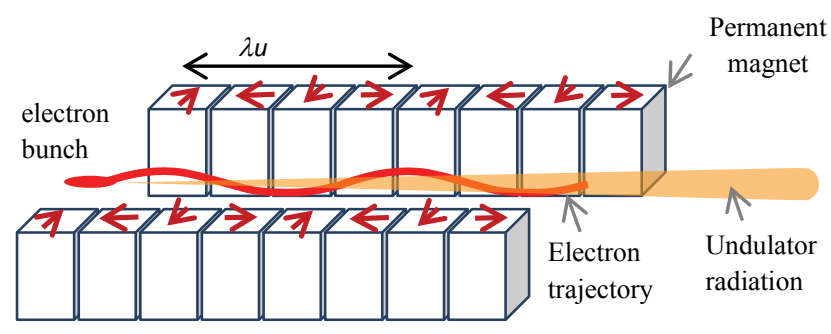

Figure 1: Schematic diagram of the vertical deflection permanent magnet Halbach type undulator.

Magnetic Chicane Bunch Compressor

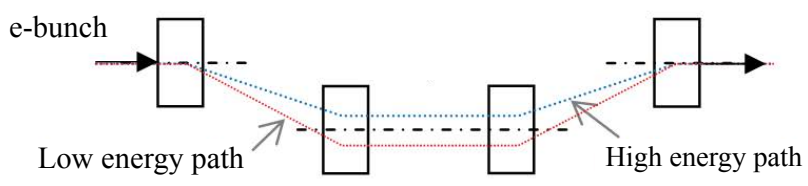

Position along the chicane:

Entrance
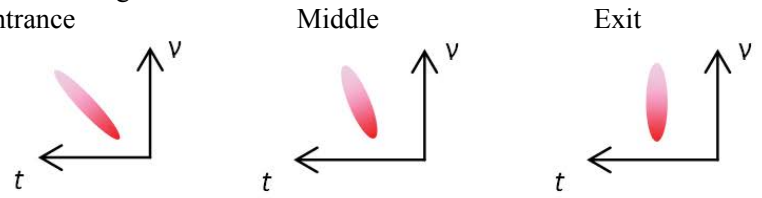

Figure 2: Schematic diagrams of the bunch compression (upper) and the longitudinal phase space evolution along the bunch compressor (lower). The head of the bunch represents by the dark shade.

This bunch compressor consists of four dipole magnets and requires the bunch with a low energy at the head and a high energy at the tail as shown in Figure 2. When such a bunch passes through the chicane, the higher energy electrons travel in the shorter path and can catch up the low energy electron at the front resulting in a shorter bunch length at the chicane exit. The suitable initial longitudinal phase space correlation of the bunch for the compression can be obtained by adjusting the laser injection phase to the RF gun. The amount of compression is determined by the first order momentum compaction factor $\left(R_{56}\right)$ which is defined as the $d s / d \gamma$ here $d s$ is the total path different and $d \gamma$ is the relative energy.

The spectral intensity of the CUR is not monochromatic due to the finite length of the CUR wave train. The wave train has the number of oscillation same as the number of periods of the undulator $\left(N_{u}\right)$ and the time duration of $T=N_{u} \lambda / c$. The spectral bandwidth $\left(\Delta \omega / \omega_{0}\right)$ of the CUR depends on the number of period of the undulator that can be calculated by

$$
\frac{\Delta \omega}{\omega_{0}}=\frac{1}{N_{u}}
$$

Where $\omega_{0}$ is the central frequency of the radiation. 


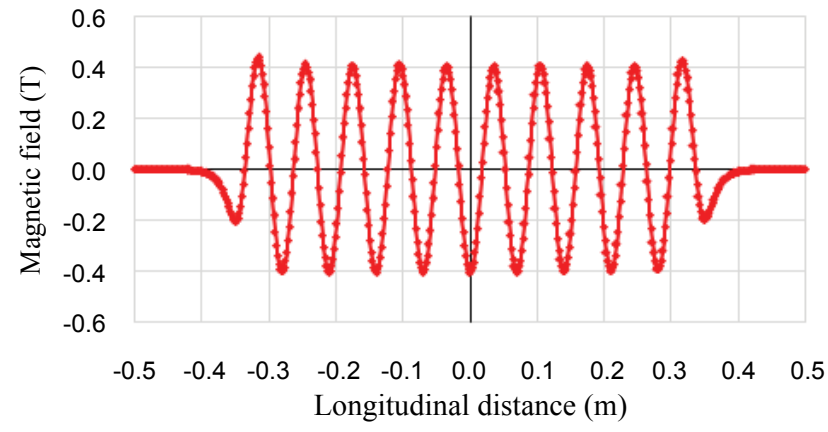

Figure 3: Measured transverse magnetic field at the center between poles at the undulator gap of $30 \mathrm{~mm}$.

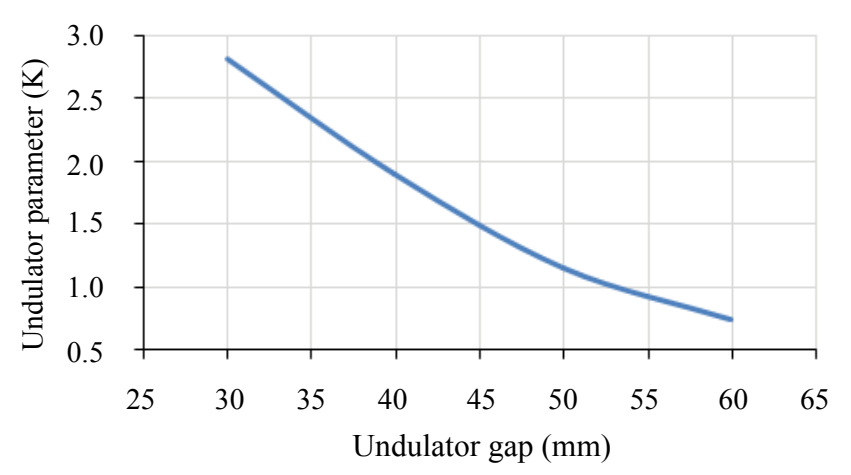

Figure 4: Undulator parameter $(K)$ as a function of undulator gap calculated from the measured magnetic field.

\section{Undulator of Compact Thz Radiation Source}

The undulator of the compact $\mathrm{THz}$ radiation source at Kyoto University is a vertical deflection planar Halbach type permanent magnet with the period length of $70 \mathrm{~mm}$ and the number of periods of 10 . The undulator gap can be manually adjusted with the minimum gap of $30 \mathrm{~mm}$ limited by the vacuum chamber. At the gap of $30 \mathrm{~mm}$, the measured peak magnetic field at the center between poles is $0.43 \mathrm{~T}$ and the maximum undulator parameter $(K)$ is 2.8 corresponding to the lowest radiation frequency of $0.17 \mathrm{THz}$. The expected spectral bandwidth is $10 \%$ for all CUR frequencies. The measurement of the transverse magnetic field at the center between poles at the undulator gap of $30 \mathrm{~mm}$ is shown in Figure 3. The calculated undulator parameter as a function of the undulator gap is shown in Figure 4.

\section{Measurement Setup}

The beam line of the compact $\mathrm{THz}$ radiation source is shown in Figure 5. The electrons were generated by injecting the $266 \mathrm{~nm}$ wavelength UV laser [11] to the copper cathode of the 1.6 cells photocathode RF gun. The laser had the repetition rate of $89.25 \mathrm{MHz}$ which was the $1 / 32$ time of the driven RF frequency $(2,856$ $\mathrm{MHz}, \mathrm{S}$-band) for the RF gun and the pulse duration at the FWHM approximately of 6 ps. The RF pulse had the

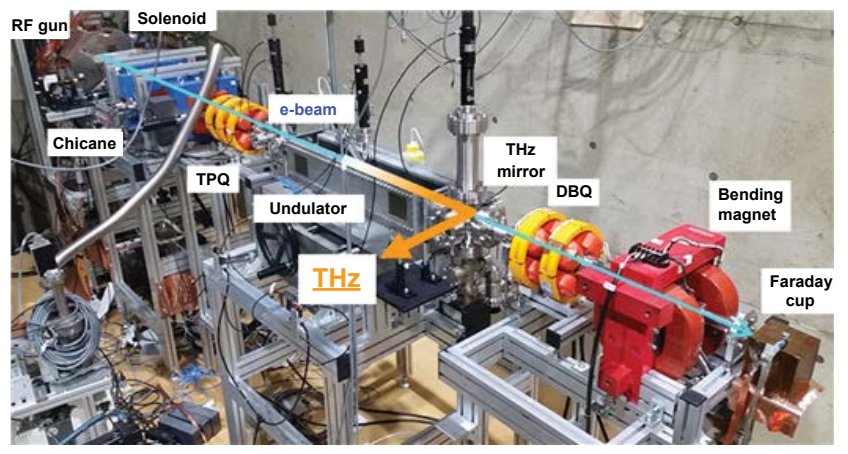

Figure 5: Beam line of the compact terahertz radiation source at Kyoto U.

macropulse repetition rate of $2 \mathrm{~Hz}$ and the time duration of $2 \mu \mathrm{s}$. The number of laser pulse was adjusted from 1 to 4 pulses per macropulse to obtain proper detected signal by detectors. This experiment operated the RF gun at the maximum power of $9 \mathrm{MW}$ that provide the beam with energy around $4.6 \mathrm{MeV}$ and energy spread of $1.3 \%$ [9]. The magnetic chicane bunch compressor operated in two conditions which were turn-on and turn-off compression. The DC current of 5.8 A supplied to the chicane dipole magnets for the turn-on compression that provided the $R_{56}$ of $-47.1 \mathrm{~mm}$. The solenoid magnet attached in front of the RF-gun, the steering magnets, the small correction coil of the chicane dipole magnets and triplet quadrupole magnets were adjusted to obtain the highest detected signal from the detectors, i.e. highest radiation intensity.

$\mathrm{THz}$ radiations from the undulator reflected out of the vacuum chamber by a retractable $\mathrm{THz}$ mirror made from a titanium film with the thickness of 50 micron installed in a 6-crosses chamber downstream the undulator. The film was attached on a circular aperture aluminum holder with the aperture diameter of $50 \mathrm{~mm}$ arranged in the 45 degree respect to the electron beam direction and 480 $\mathrm{mm}$ apart from the middle of the undulator. The fused silica with the aperture diameter $65 \mathrm{~mm}$ was employed as a $\mathrm{THz}$ window and the distance from the mirror to the window was about $120 \mathrm{~mm}$. Two types of small-size uncooled detectors were used for the $\mathrm{THz}$ radiation detection. First, a Schottky Zero-Bias Barrier Diode (SBD) detector (Millitech, DET-05-RPFW0) has the sensitivity of higher than $250 \mathrm{mV} / \mathrm{mW}$ [12] in the frequency range from 140 to $220 \mathrm{GHz}$. The SBD detector was installed inside the rectangular wave guide, therefore, it could pick the signal in one direction of polarization. Second, a pyroelectric detector (PHLUXi, PYD-1-018) equipped with the built-in lens and the visible light filter. The detector has not been calibrated in the $\mathrm{THz}$ frequency range. The signals from the detectors were recorded by $1 \mathrm{GHz}$ oscilloscope (Textronics, DPO4104). The bunch charge was measured by a $10 \mathrm{~cm}$ cubic out-of-vacuum carbon 
Table 1: Parameters of the undulator radiation measurement.

\begin{tabular}{|l|l|}
\hline Parameters & Values \\
\hline Beam energy & $4.6 \mathrm{MeV}$ \\
\hline Energy spread & $1.3 \%$ \\
\hline Laser pulse duration & $6 \mathrm{ps}$ (FWHM) \\
\hline Laser size at cathode (Hor./Ner.) & $0.4 / 0.5 \mathrm{~mm} \mathrm{rms}$ \\
\hline Laser distribution (Hor./Ver.) & Gaussian/Gaussian \\
\hline Number of laser pulse per macropulse & 1 to 4 pulses \\
\hline Solenoid magnetic field & 163 to $194 \mathrm{mT}$ \\
\hline$R_{56}$ of the magnetic chicane & $0,-47.1 \mathrm{~mm}$ \\
\hline Undulator gap & 30 to $55 \mathrm{~mm}$ \\
\hline
\end{tabular}

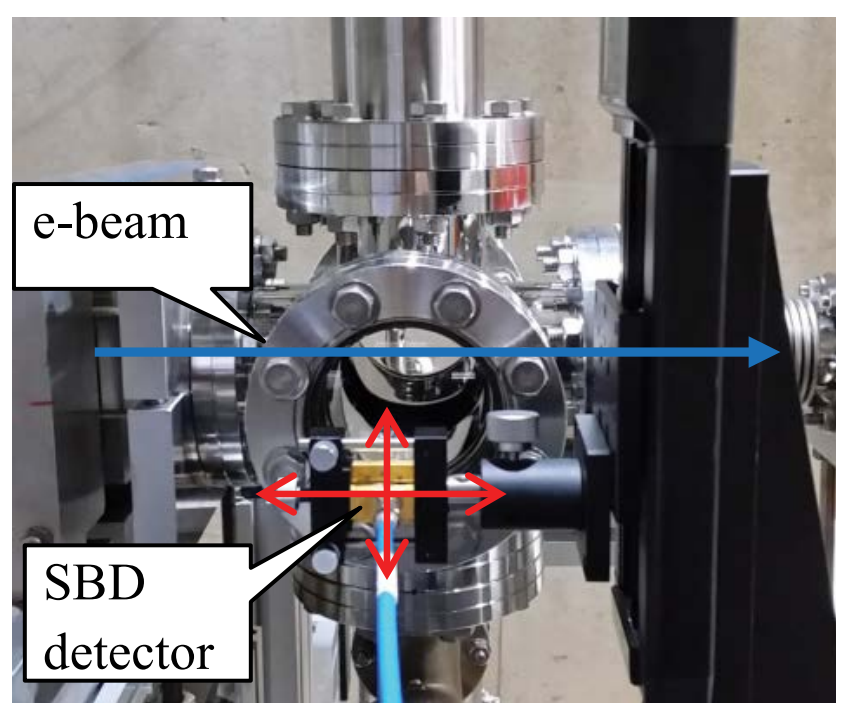

Figure 6: Set up of undulator spatial distribution measurements. The THz mirror was move-up condition.

Faraday cup placed at the end of the beam line and the signal was read by $200 \mathrm{MHz}$ oscilloscope (Iwatsu, DS5624A). The lead blocks were put beside the beam line to prevent bremsstrahlung radiation inducing noise in the detector. The beam line control and measurements were performed under the LABVIEW environment. The parameters for the undulator radiation measurement are listed in Table 1.

\section{Spatial distribution}

The spatial distributions of the undulator radiation were measured by scanning the SBD detector in two-dimensions without focusing optics. The scanning plane was normal to the extracted $\mathrm{THz}$ beam with the distance approximately of $140 \mathrm{~mm}$ from the center of the $\mathrm{THz}$ mirror. The detector was mounted on two translational stages (Sigmakoki, SG SP 20-85) and scanned with $4 \mathrm{~mm}$ interval in both horizontal and vertical directions. The measurement set up of the spatial distribution of the undulator radiation is shown in Figure 6.

\section{Total intensity}

The total intensity was measured by using a focusing optics to focus whole extracted beam to the pyroelectric

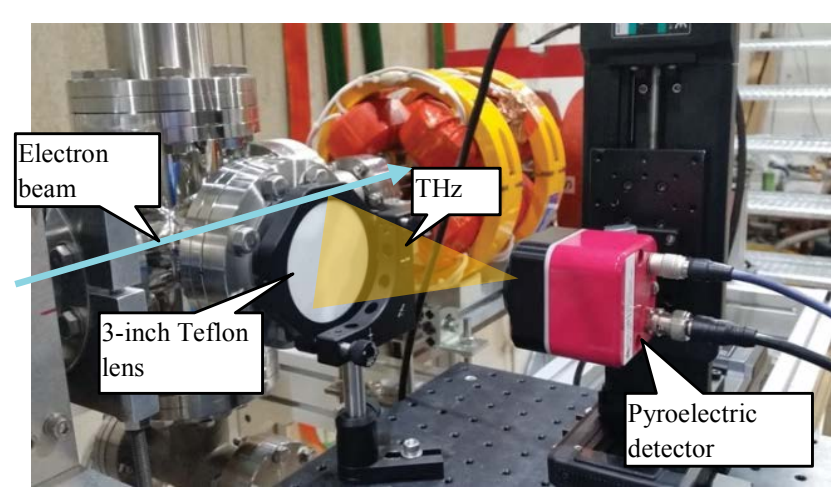

Figure 7: Total intensity measurement of the undulator radiation.

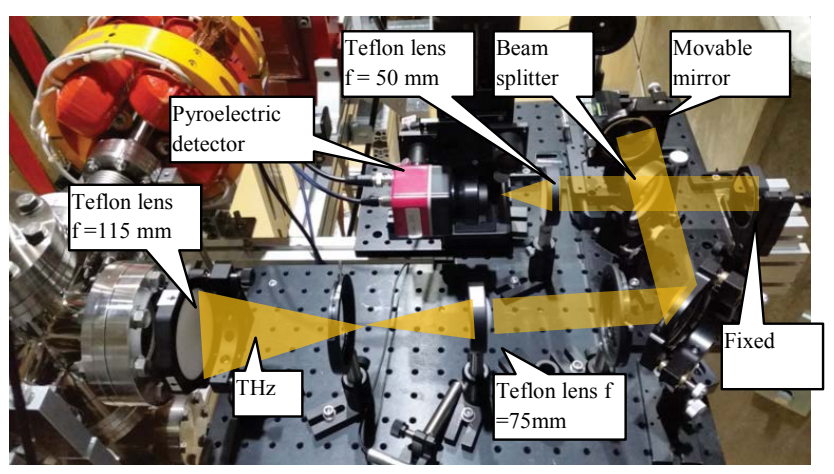

Figure 8: Set up of the Michelson interferometer for the power spectrum measurement.

detector. A plano-convex polytetrafluoroethylene lens or Teflon lens (Thorlabs, LAT115) with the 3 inch diameter and the focal length of $115 \mathrm{~mm}$ was employed and placed next to the $\mathrm{THz}$ window. The detector was aligned to the focal point by means of the translation stage. The setup of the total intensity measurement is shown in Figure 7.

\section{Power spectrum}

The setup of an in-air Michelson interferometer for the power spectrum measurement is shown in Figure 8. Two focusing lens which were the 3-inch and 2-inch diameter Teflon lens (Thorlabs, LAT115, LAT75) with the focal length of 115 and $75 \mathrm{~mm}$ were utilized for reducing the $\mathrm{THz}$ beam size suitable for the interferometer. The fixed and movable mirrors of the interferometer were 2-inch gold-coated flat mirror (Thorlabs, PF20-03-M01). For measuring the interferogram, the movable mirror position was varied from -8 to $8 \mathrm{~mm}$ with the step size of $0.1 \mathrm{~mm}$. The total optical path different was $32 \mathrm{~mm}$ and the time window of the measurement was $106.7 \mathrm{ps}$ and the frequency resolution, which is the inverse of the time window, was $9.37 \mathrm{GHz}$.

Two types of the beam splitter which were a $100 \mathrm{mi}-$ cron thick sapphire beam splitter and an inconel coated pellicle beam splitter with the thickness of 2 micron were used. Due to the efficiency drop of the sapphire 
(a)

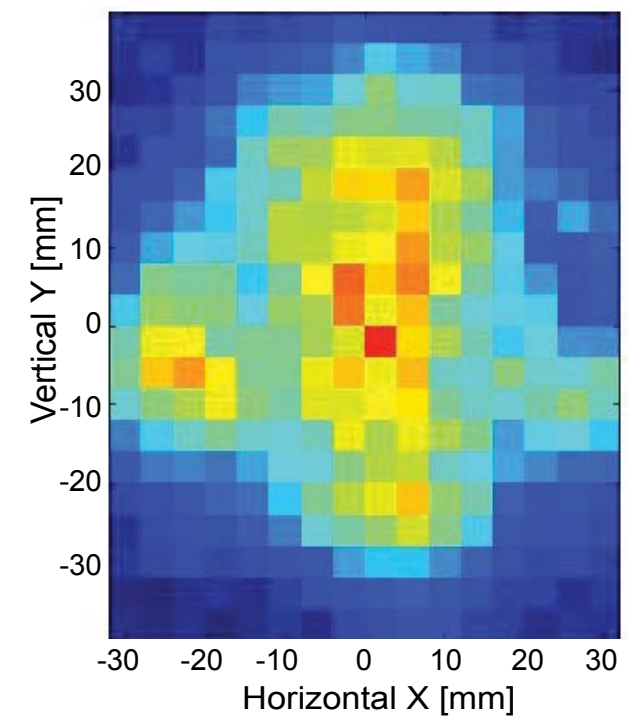

(c)

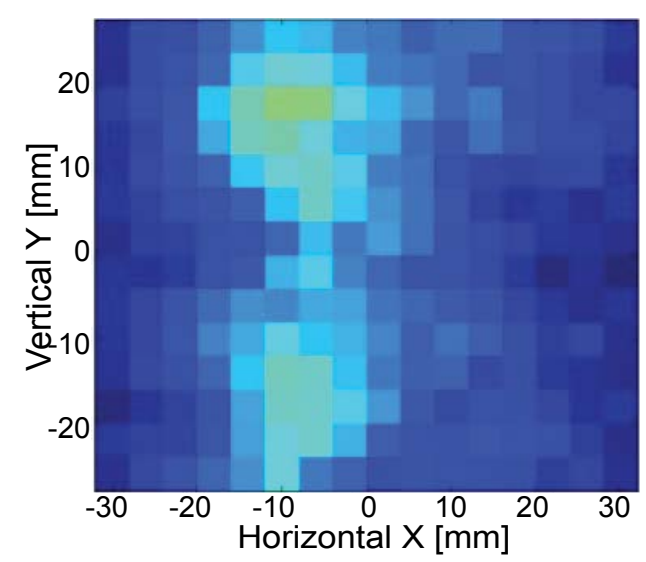

(b)

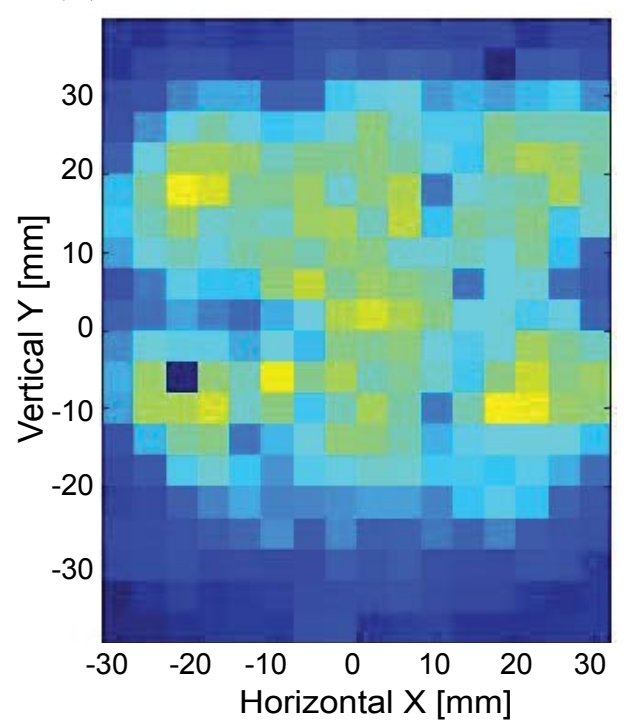

(d)

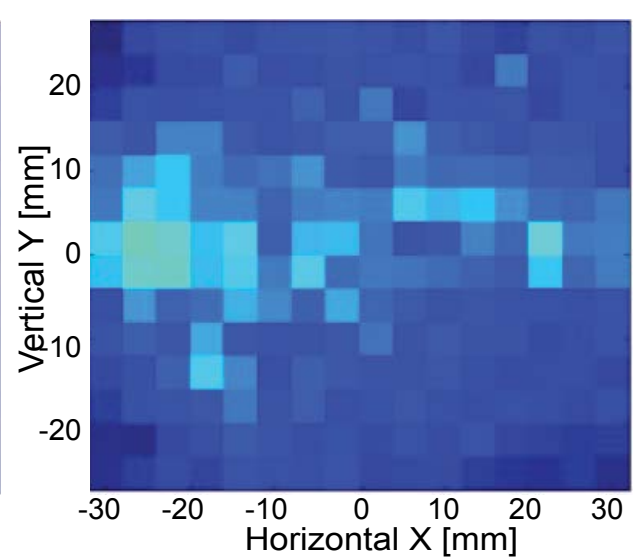

Figure 9: Spatial distributions of the CUR of the compressed bunch at the undulator gap of a) $30 \mathrm{~mm}$; b) $35 \mathrm{~mm}$; c) $180 \mathrm{~mm}$ and the uncompressed bunch at the undulator gap of d) $30 \mathrm{~mm}$.

beam splitter at around $0.5 \mathrm{THz}$, this beam splitter was used for the measurement at the frequency less than 0.4 $\mathrm{THz}$ while the inconel coated beam splitter was used at the frequency more than $0.4 \mathrm{THz}$. The combined beams exiting the interferometer were focused by the 2-inch Teflon lens with the focal length of $50 \mathrm{~mm}$ (Thorlabs, LAT50). The pyroelectric detector with the built-in lens was mounted on the translation stages and aligned to the focusing point of the combined beam.

\section{Measurement Result}

\section{Spatial distribution}

The spatial distribution measurements were performed at the laser injection phase of $20 \mathrm{deg}$ and the $R_{56}$ of $-47.1 \mathrm{~mm}$ which provided the best compression condition as studied in [9]. The undulator gap was varied to 30,35 and 180 which were the minimum gap, the gap at the highest frequency response of the SBD detector and the largest gap. These gaps had the undulator pa- rameter $(K)$ of $2.8,2.3$ and 0 and the calculated central frequencies of the radiation $\left(\omega_{0}\right)$ of $0.17,0.23$ and 0.86 $\mathrm{THz}$, respectively. The number and the energy of the laser pulse per macro pulse were 4 pulses and $300 \mu$ J. The results of the spatial distribution of the undulator radiation are shown in Figure 9. Note that all shown results were the radiation with the vertical polarization which is the same direction as the deflection of electron inside the undulator. The radiation with the horizontal polarization was also measured but the signal was very weak. As the results, the radiation from the compressed bunch concentrated in the narrow cone at the beam center and aligned to the center of the $\mathrm{THz}$ mirror (Figure 9a). The radiation from the uncompressed bunch was weak (Figure 9d). At the gap of 30 and $35 \mathrm{~mm}$, the calculated opening angle $(K / \gamma)$ of the radiation cone corresponding to the beam energy of $4.6 \mathrm{MeV}(\gamma \approx 10)$ were 16 and 13.1 deg that larger than the acceptance angle of the mirror at around $6 \mathrm{deg}$. Therefore, only the center part of the radi- 
ation could be reflected by the mirror. But the other part of the radiation might be reflected by the internal side of the 6-cross chamber as clearly seen in Figure 9b. In case

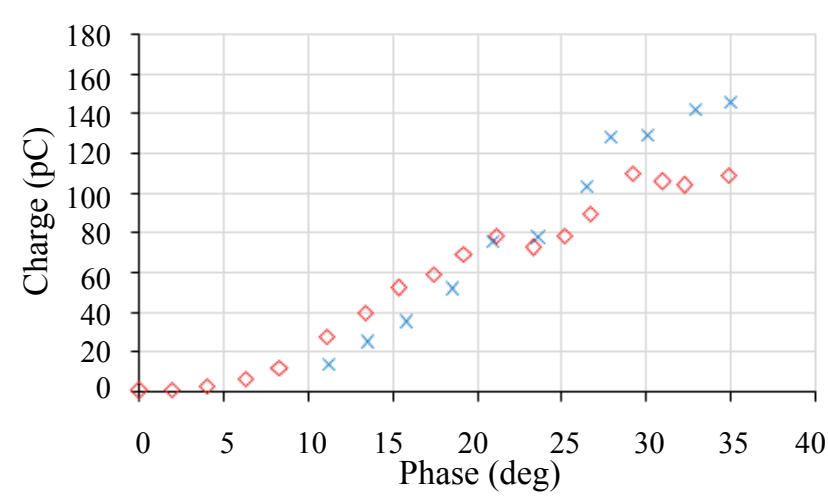

Figure 10: Measured bunch charge as a function of the laser injection phase at the undulator gap of $30 \mathrm{~mm}$ (square marks: compressed bunch, cross marks: uncompressed bunch).
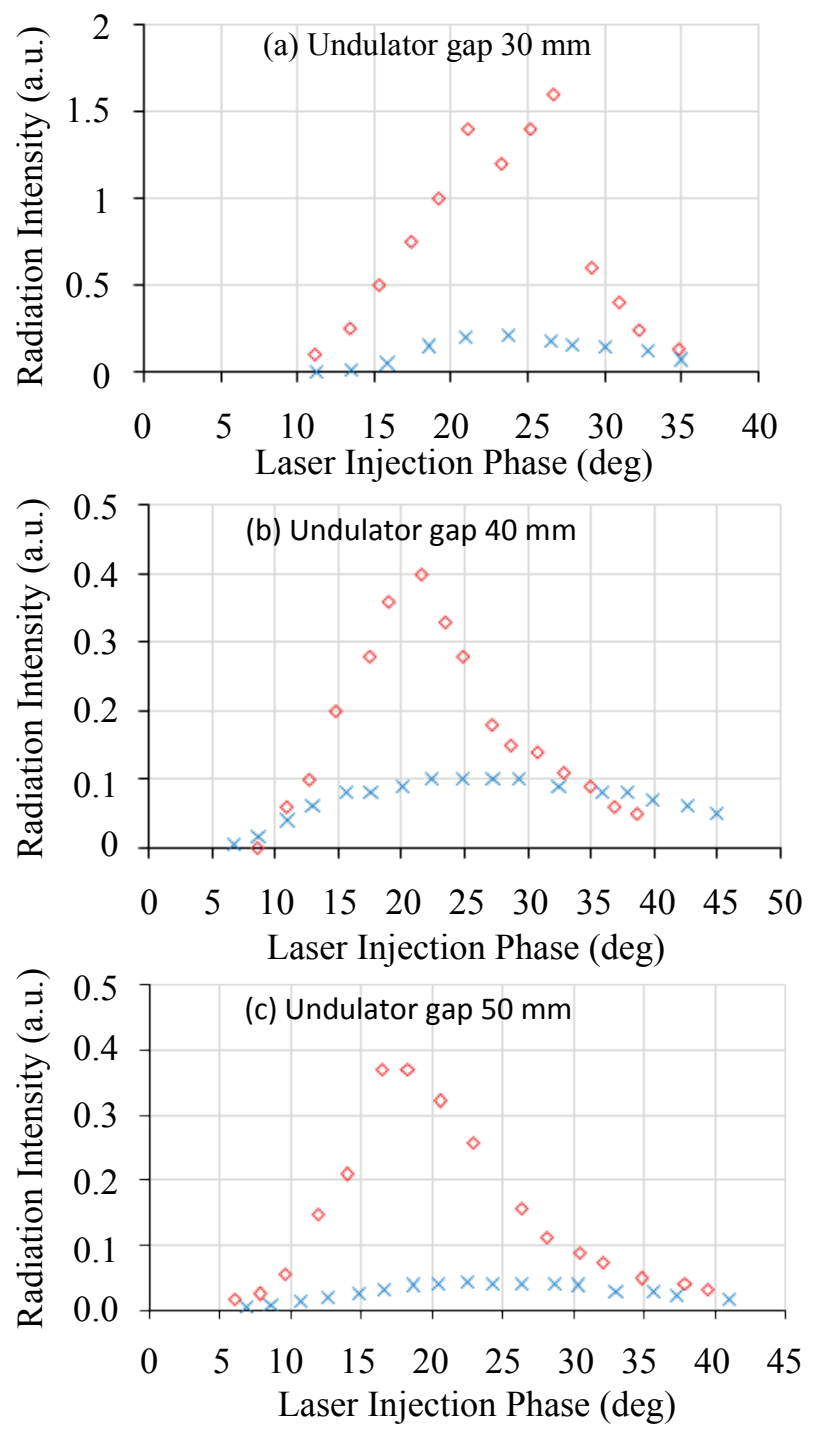

Figure 11: CUR intensity dependence on the laser injection phase at the undulator gap of a) $30 \mathrm{~mm}$; b) $40 \mathrm{~mm}$ and c) 50 $\mathrm{mm}$ (square marks: compressed bunch, cross marks: uncompressed bunch). of the largest undulator gap at $180 \mathrm{~mm}$ (Figure 9c), the CUR could not be observed but the detected radiation was a coherent transition radiation, which was emitted at the surface of $\mathrm{THz}$ mirror made of Ti film when electron bunch was injected to the film.

\section{Total intensity}

The measured bunch charge and the radiation intensities dependence on the laser injection phases of the uncompressed and compressed bunch are shown in Figure 10 and Figure 11. The measurement was performed with the number and the energy of the laser pulse per macropulse of 4 pulses and $300 \mu \mathrm{J}$, respectively. The measured bunch charge of both compression conditions had almost the same for a given laser injection phase and undulator gap. But the CUR intensity of the compressed bunch was significantly higher than the uncompressed bunch. It means that the chicane successfully compressed the bunch and enhanced the form factor resulting in a high CUR intensity. The peak intensity occurred at the phase around 15 to 25 degrees, where the bunch had suitable initial longitudinal phase space correlated with the compression at the $R_{56}$ of $-47.1 \mathrm{~mm}$. The factor of the intensity enhancement reached up to 8.3 at the undulator gap of $50 \mathrm{~mm}$. The CUR from the larger undulator gap had smaller intensity compared to the narrower one, because the form factor of the same bunch became smaller at a higher frequency.

\section{Power spectrum}

The autocorrelation intensity dependencies as a function of path different or interferograms measured by the Michelson interferometer for the undulator gap from 30 to $55 \mathrm{~mm}$ are shown in Figure 12. Note that the interferograms are shifted in vertical for easier seeing the lines. The measurement was performed only for the compressed bunch because the signal had strong enough to detect. The undulator radiation spectra calculated by Fast Fourier Transform corresponding to the interferograms are shown in Figure 13. As the result, the central

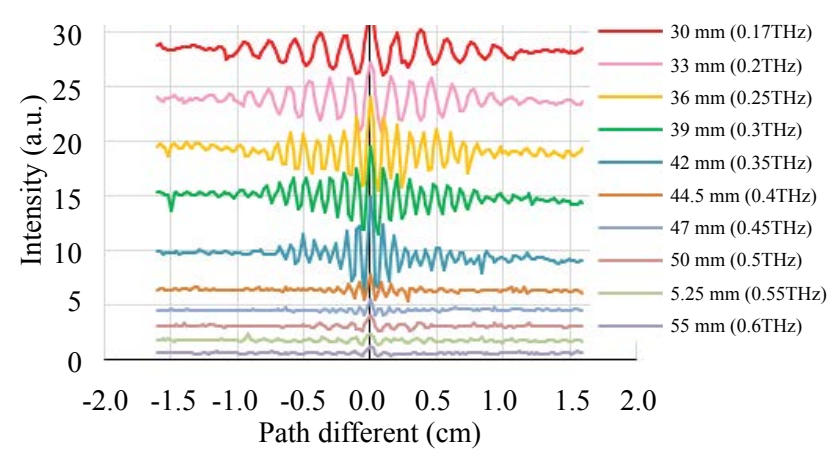

Figure 12: Measured interferograms by the Michelson interferometer for the compressed bunch with the different undulator gaps. 


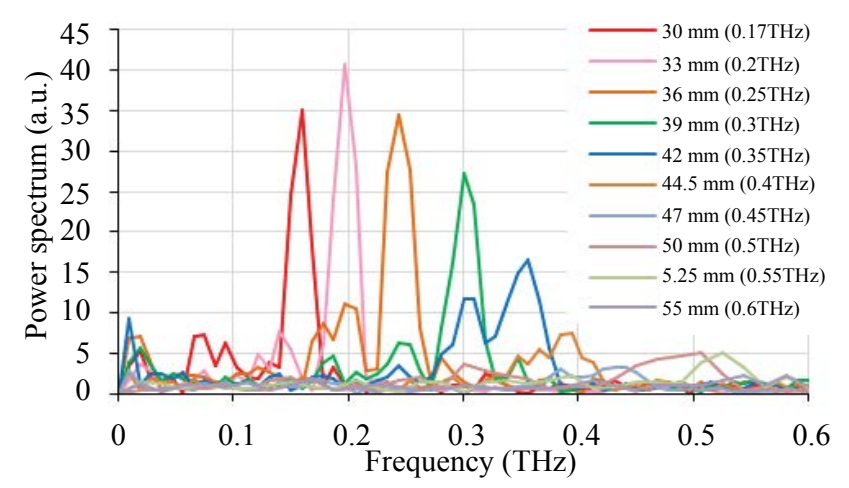

Figure 13: Power spectrum of the CUR for the different undulator gaps 30 to $55 \mathrm{~mm}$. The frequencies in the bracket of the legend are the calculated radiation frequency.

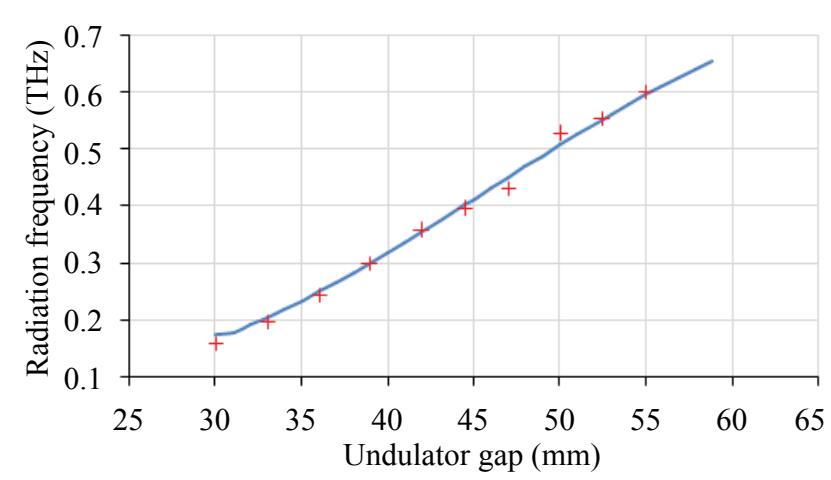

Figure 14: Central frequency of the CUR by calculation (solid line) and measurement (cross mark).

frequency was in the range from 0.17 to $0.55 \mathrm{THz}$ and the FWHM bandwidth was approximately $10 \%$. The central frequencies by the measurement had a good agreement with the calculation and are shown in Figure 14. The spectra were not monochromatic and contained smaller component beside the central frequency. The highest frequency that could be distinguished from the noise floor was $0.55 \mathrm{THz}$.

\section{Conclusion}

The accelerator based compact terahertz radiation source has been successfully developed and the first terahertz coherent undulator radiation has been observed. Some measurements have been carried out to characterize the radiation properties. The system could generate the radiation in the frequency range from 0.17 to 0.55 $\mathrm{THz}$ with the bandwidth approximately $10 \%$ from the low energy electron beam of $4.6 \mathrm{MeV}$. The radiation was coherent and the intensity could be enhanced by the magnetic chicane bunch compressor. For the compressed bunch at the $R_{56}$ of $-47.1 \mathrm{~mm}$, the radiation intensity had the peak at the laser injection phase of 15 to 25 degrees. The factor of the intensity enhancement reached maximum at 8.3 . The absolute value of intensity was not calculated yet. The calibration of the detectors and the optical component are still under investigation.

\section{References}

1. M Tonouchi (2007) Cutting edge terahertz technology. Nat Photon 1: 97-105.

2. GR Neil, GL Carr, Joseph F Gubeli, K Jordan, Michael C Martin, et al. (2003) Production of high power femtosecond terahertz radiation. Nucl Instr Meth Phys Res A 507: 537-540.

3. M Gensch, L Bittner, A Chesnov, H Delsim-Hashemi, M Drescher, et al. (2008) New infrared undulator beamline at FLASH. Infrared Phys Technol 51: 423-425.

4. Z Wu, Fisher AS, Goodfellow J, Fuchs M, Daranciang D, et al. (2013) Intense terahertz pulses from SLAC electron beams using coherent transition radiation. Rev Sci Instrum 84: 022701.

5. A Doria, Gallerano GP, Giovenale E, Messina G, Spassovsky I (2004) Enhanced coherent emission of $\mathrm{THz}$ radiation by energy-phase correlation in a bunched electron beam. Phys Rev Lett 93: 264801.

6. X Wen, Senlin Huang, Lin Lin, Fang Wang, Feng Zhu, et al. (2016) Superradiant THz undulator radiation source based on a superconducting photo-injector. Nucl Instr Meth Phys Res A 820: 75-79.

7. H Zen, Sikharin Suphakul, Toshiteru Kii, Kai Masuda, Hideaki Ohgaki (2016) Present Status and Perspective of Long Wavelength Free Electron Laser at Kyoto University. Physics Procedia 84: 47-53.

8. K Damminsek, S Rimjaem, C Thongbai (2015) Electron beam properties from a compact seeded terahertz amplifier at Kyoto University. Proceedings of FEL2015, Daejeon, Korea, 85-88.

9. S Suphakul, H Zen, K Morita, K Torgasin, K Masuda, et al. (2016) Generation of Short Bunch Electron Beam from Compact Accelerator for Terahertz Radiation. Proceedings of IPAC2016, Busan, Korea, 1757-1759.

10. D Bocek (1995) Generation and Characterization of Supper radiant Undulator Radiation. SLAC-PUB-7016.

11. H Zen, S Suphakul, T Kii, H Ohgaki (2015) Development of Photocathode Drive Laser System for RF Guns in KU-FEL. Proceedings of FEL2014, Basel, Switzerland, 828-831.

12. http://www.millitech.com/MMW-MixerDetector-DET.htm 